\title{
Global-in-Time Asymptotic Solutions to Kolmogorov-Feller-Type Parabolic Pseudodifferential Equations with Small Parameter-Forward- and Backward-in-Time Motion
}

\author{
V. G. Danilov \\ Moscow Institute of Electronics and Mathematics, National Research University "Higher School of Economics", \\ B. Trekhsvyatitel'skii Per. 3, Moscow 109028, Russia \\ Correspondence should be addressed to V. G. Danilov; vgdanilov@mail.ru
}

Received 31 January 2013; Accepted 2 April 2013

Academic Editor: Allan Peterson

Copyright (c) 2013 V. G. Danilov. This is an open access article distributed under the Creative Commons Attribution License, which permits unrestricted use, distribution, and reproduction in any medium, provided the original work is properly cited.

We discuss the construction of solutions to the inverse Cauchy problem by using characteristics.

\section{Forward-in-Time Motion}

The goal of the present paper is to present a new approach to the construction of asymptotic (approximating) solutions for parabolic PDE by using bicharacteristics. This approach allows one to construct global-in-time solutions not only to the usual Cauchy problems, but also for the inverse problems. We will work with Kolmogorov-Feller-type equations with diffusion and jump terms. The equation under consideration has the form:

$$
\begin{aligned}
& -\varepsilon \frac{\partial u}{\partial t}+P\left({ }_{x}^{2},-\varepsilon \frac{\partial}{\partial x}\right) u=0, \\
& \left.u(x, t, \varepsilon)\right|_{t=0}=e^{-S_{0}(x) / \varepsilon} \varphi^{0}(x),
\end{aligned}
$$

where $P(x, \xi)$ is the symbol of the Kolmogorov-Feller operator and $\varepsilon \rightarrow+0$ is a small parameter characterizing the frequency and the amplitude of jumps of the Markov stochastic process with transition probability given by $P(x, \xi)$. To be more precise, we will keep in mind the following form of $P(x, \xi)$ :

$$
\begin{aligned}
P(x, \xi)= & (A(x) \xi, \xi)+(B(x), \xi) \\
& +\int_{\mathbb{R}^{n}}\left(e^{i(\xi, \nu)}-1\right) \mu(x, d \nu),
\end{aligned}
$$

where $A(x)$ is a positive smooth matrix and $\mu(x, d \nu)$ is a family of positive bounded measures smooth in $x$ such that

$$
\int_{\mathbb{R}^{n}} v_{i} \mu(x, d v)=0, \quad i=1, \ldots, n,
$$

and $B(x)$ is smooth in $x$. The construction of a forward-intime global asymptotic solution to equations of this type was developed by Maslov, [1-3]; for a version of this construction, see also [4-6]. Maslov's approach is based on ideas similar to the ideas used in his famous canonical operator construction (or in the later and similar theory of Fourier integral operators). This construction is based on some integral representations and cannot be used to construct backward-in-time solutions. Another approach to the construction of global asymptotic solutions was suggested in [7] and is based on the construction of generalized solutions to the continuity equation in a discontinuous velocity field [8].

In the present paper, we develop an algorithm for constructing asymptotic solutions. Some steps of this algorithm are rigorously justified only in the one-dimensional case, but all the required facts can be checked "experimentally," while the problem is considered in the multidimensional case. Therefore, we give general formulations but sometimes prove them only in the one-dimensional case. 
We assume that the solutions under consideration admit the following limits:

(1) the logarithmic pointwise $\operatorname{limit}_{\lim _{\varepsilon \rightarrow 0}}(-\varepsilon \ln u)$. We denote this limit by $S=S(x, t)$ and assume that it is a piecewise smooth function with bounded first derivatives and singular support in the form of a stratified manifold $M$.

(2) the weak limit of the expression $\exp (2 S / \varepsilon) u^{2}$. We denote it by $\rho$ and assume that $\rho$ is the sum of a function smooth outside $M\left(\rho_{\text {reg }}\right)$ and the Dirac $\delta$ function on $M$. Note that here we deal with the limit in the weighted weak sense.

If $S$ and $\rho$ are smooth functions, then the following representation is true (in the usual sense):

$$
u=\exp \left(-\frac{S}{\varepsilon}\right) \sqrt{\rho}_{\mathrm{reg}}(1+o(\varepsilon))
$$

Definition 1. A solution to the Cauchy problem under consideration is called a generalized WKB solution if it satisfies conditions (1) and (2).

Example 2. One can see that if

$$
\left.u\right|_{t=0}=\exp \left(-\frac{S_{0}}{\varepsilon}\right) \varphi^{0}(x),
$$

where $S_{0} \geq 0$ is a smooth function, $\varphi^{0} \in C_{0}^{\infty}$, then the WKBlike approach can be used (Kifer, [9]; Maslov, [1-3]). This gives an asymptotic (approximating) solution of the form (cf. [10])

$$
\begin{aligned}
u_{\mathrm{as}} & =u_{\mathrm{as}}(x, t, \varepsilon) \\
& =\exp \left(-\frac{S(x, t)}{\varepsilon}\right)\left(\varphi_{0}(x, t)+\cdots+\varepsilon^{k} \varphi_{k}(x, t)\right)
\end{aligned}
$$

for arbitrary $k$. Here, $S(x, t)$ is the solution to the Cauchy problem for the Hamilton-Jacobi equation

$$
S_{t}+P(x, \nabla S)=0, \quad S_{t=0}=S_{0}(x),
$$

and $\varphi_{0}=\varphi_{0}(x, t)$ is the solution to the transport equation

$$
\begin{gathered}
\varphi_{0 t}+\left(\nabla_{\xi} P(x, \nabla S), \nabla \varphi_{0}\right)+\frac{\varphi_{0}}{2} \operatorname{tr}\left(P_{\xi \xi}(x, \nabla S) S_{x x}\right)=0 \\
S_{t=0}=S_{0}(x)
\end{gathered}
$$

Both of the solutions $S$ and $\varphi_{0}$ are defined via solutions of the Hamiltonian system

$$
\begin{gathered}
\dot{x}=\nabla_{\xi} P(x, p),\left.\quad x\right|_{t=0}=\alpha, \\
\dot{p}=-\nabla_{x} P(x, p),\left.\quad p\right|_{t=0}=\nabla S_{0}(\alpha) .
\end{gathered}
$$

They are smooth, while

$$
\frac{D x}{D \alpha} \neq 0 \text {. }
$$

There are symplectic geometry objects corresponding to this construction:
(1) the phase space $\mathbb{R}_{x, p}^{2 n}=\mathbb{R}_{x}^{n} \times \mathbb{R}_{p}^{n}$,

(2) a Lagrangian manifold $\Lambda_{n}^{t} \in \mathbb{R}_{x, p}^{2 n}$,

$$
\Lambda_{n}^{0}=\left(x=\alpha, p=\nabla S_{0}(\alpha)\right), \quad \Lambda_{n}^{t}=g_{P}^{t} \Lambda_{n}^{0},
$$

where $g_{P}^{t}$ is a shift mapping along the Hamiltonian system trajectories,

(3) the projection mapping $\pi: \Lambda_{n}^{t} \rightarrow \mathbb{R}_{x}^{n}$ with Jacobi matrix $\partial x / \partial \alpha$. one.

The main assumption that we must prove is the following

The Hamiltonian system trajectories form a fibration of the phase space (at least in the area of the phase space where we are working).

Let $D x / D \alpha \neq 0$ for $t \in[0, T]$, then we have the following statement (Maslov, [1, 2]; see also Danilov, [4-6]).

Theorem 3. The inequality

$$
\operatorname{Re} P(x, p+i \eta) \leq P(x, p), \quad \eta \in \mathbb{R}^{n}
$$

is necessary and sufficient for the estimate

$$
\left\|\exp \left(\frac{S(x, t)}{\varepsilon}\right)\left(u_{\text {as }}-u\right)\right\|_{C\left(\mathbb{R}_{x}^{n}\right)} \leq C_{M} \varepsilon^{M},
$$

where $M=M(k) \rightarrow \infty$ as $k \rightarrow \infty$ and the function $S$ is a solution to the Hamilton-Jacobi equation with Hamiltonian $P(x, p)$.

It is easy to verify that the function $P=P(x, \xi)$-the symbol introduced above-satisfies the inequality mentioned in the theorem.

Example 4. In the special case corresponding to Example 2, we have

$$
-\varepsilon u_{t}+\varepsilon^{2} u_{x x}=0,\left.\quad u\right|_{t=0}=\exp \left(-\frac{S_{0}}{\varepsilon}\right) \varphi^{0} .
$$

The corresponding system is

$$
\begin{gathered}
S_{t}+\left(S_{x}\right)^{2}=0, \quad(\text { Hamilton-Jacobi equation) }, \\
\varphi_{0 t}+2 S_{x} \varphi_{0 x}+S_{x x} \varphi_{0}=0, \quad \text { (transport equation) }, \\
\dot{x}=2 p,\left.\quad x\right|_{t=0}=\alpha, \quad \text { (Hamiltonian system). } \\
\dot{p}=0,\left.\quad p\right|_{t=0}=\frac{\partial S_{0}}{\partial \alpha}, \quad
\end{gathered}
$$

Its solution has the form

$$
\begin{gathered}
x=\alpha+2 t \frac{\partial S_{0}}{\partial \alpha}, \quad p=\left.p\right|_{t=0}, \\
\frac{D x}{D \alpha}=1+2 t \frac{\partial^{2} S_{0}}{\partial \alpha^{2}} .
\end{gathered}
$$

We have two different cases, see Figures 1 and 2. 


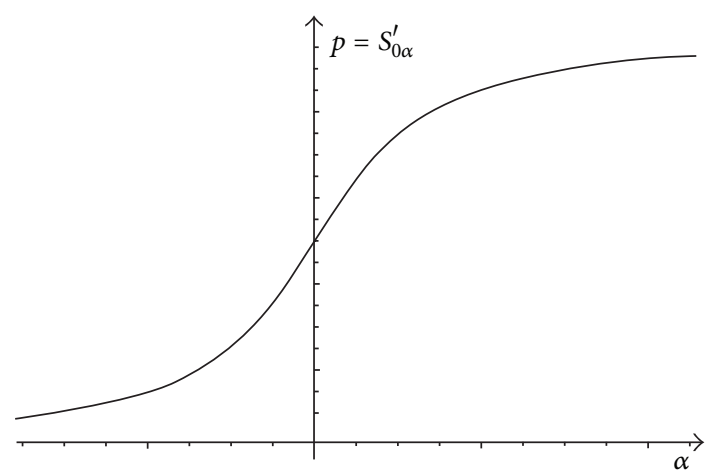

FIGURE 1: Solution $\alpha=\alpha(x, t)$ exists for all $t$.

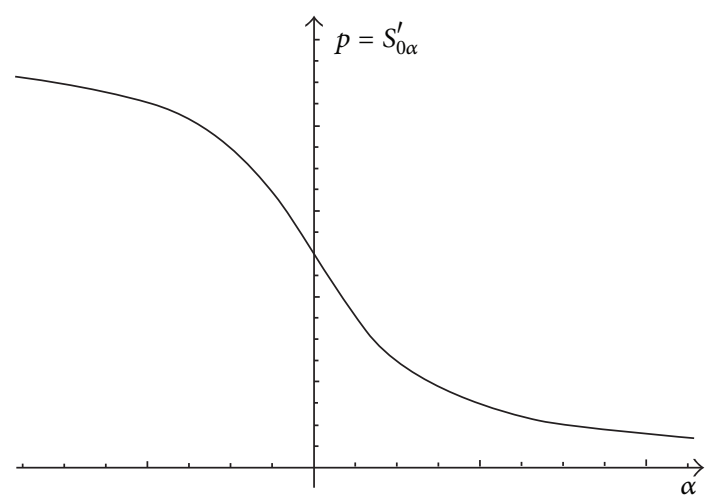

FIgURE 2: Solution $\alpha=\alpha(x, t)$ exists for $t<t^{*}=\max \left|2 S_{0 \alpha \alpha}^{\prime \prime}\right|^{-1}$.

For $t>t^{*}$ in case corresponding to Figure 2 we get $\Lambda_{t}^{1}$ of the shape plotted in Figure 3.

One can see that there are three values of $S$ at the point $\bar{x}$ in the last case. This means that we can present an asymptotic solution near this point as the linear combination

$$
u=\sum_{j=1}^{3} c_{j} u_{j}
$$

where each of the functions $u_{j}=\exp \left(-S_{j} / \varepsilon\right) \varphi_{j}$, and $j=$ $1,2,3$, satisfy the equation with the same accuracy. But the functions themselves are not equivalent in contrast to the hyperbolic case.

For example, it is clear that if the inequality

$$
S_{1}(\bar{x}, t)>S_{2}(\bar{x}, t)
$$

holds at a certain point $\bar{x}$, then the "WKB" solutions $u_{1}$ and $u_{2}$ at the point $\bar{x}$ satisfy the relation

$$
\begin{aligned}
\left.u_{1}\right|_{x=\bar{x}} & =e^{-S_{1}(\bar{x}, t) / \varepsilon} \varphi_{1}(\bar{x}, t) \\
& =\left.e^{-S_{1}(\bar{x}, t) / \varepsilon} \varphi_{2}(\bar{x}, t)\left(\frac{e^{-\left(S_{1}-S_{2}\right) / \varepsilon} \varphi_{1}}{\varphi_{2}}\right)\right|_{x=\bar{x}} \\
& =\left.u_{2}\right|_{x=\bar{x}} O\left(\varepsilon^{N}\right),
\end{aligned}
$$

where $N>0$ is an arbitrary number. This follows from the fact that the difference $\left.\left(S_{1}-S_{2}\right)\right|_{\bar{x}}$ in parentheses in the exponent is positive.

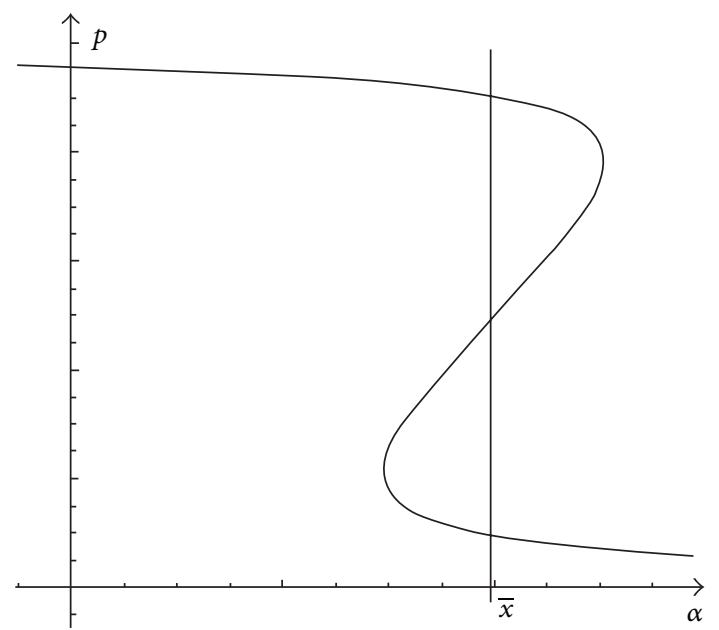

FIgURe 3: Lagrangian curve for $t>t^{*}$.

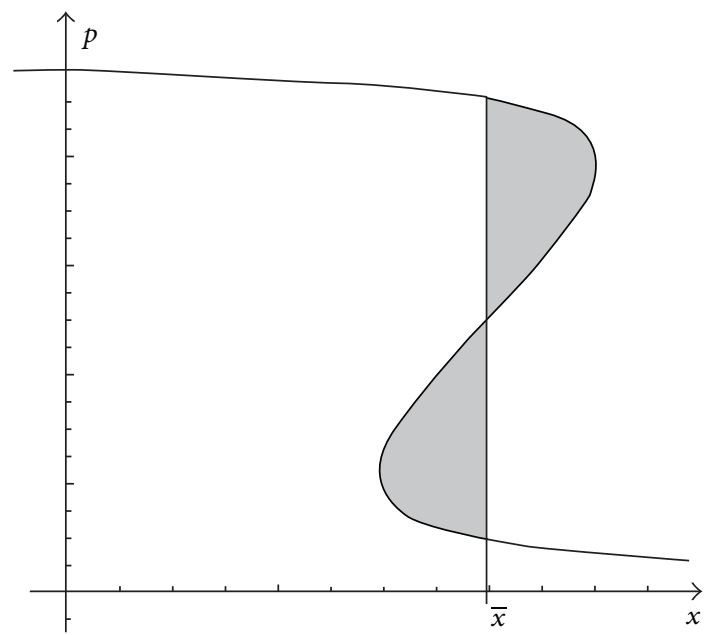

Figure 4

Thus, at each point in formula (17), it is necessary to choose the term, where the function $S_{j}$ is minimal. Such a choice leads to an expression of the form

$$
u=e^{-\Phi(x, t) / \varepsilon} \varphi(x, t),
$$

where $\Phi=\Phi(x, t)=\min _{x}\left\{S_{j}(x, t)\right\}$. It is clear that the expression (20) is the leading term of the approximate solution.

The corresponding Lagrangian manifold is shown in Figure 4.

The vertical line is located so that the dashed squares are equal to each other; see Figure 4.

It is interesting to note that, in the one-dimensional case, there is a direct connection between the Hamilton-Jacobi equation

$$
S_{t}+H\left(x, S_{x}\right)=0
$$

and conservation law of the form

$$
u_{t}+\frac{\partial}{\partial x} H(x, u)=0,
$$


where $u=S_{x}$ and the velocity of the vertical line is defined by the Rankine-Hugoniot condition corresponding to the conservation law. If the Lagrangian manifold has a jump, then the first derivative of the corresponding value function

$$
\Phi(x, t)=\min _{x} S(x, t)
$$

also has a jump.

Thus, we can state that the Lagrangian manifold is a key point for construction and investigation of asymptotic solutions.

It follows from the real analyticity assumption for all objects that there is no "concentration of singularities," that is, each of them can be considered separately in a certain sense, and one can construct a value function as a solution of the Hamilton-Jacobi-Bellman equation.

What about the amplitude function? As was mentioned above, it is a solution of the transport equation

$$
\varphi_{0 t}+\left(\dot{x}, \nabla \varphi_{0}\right)+\frac{\varphi_{0}}{2} \operatorname{tr}\left(P_{\xi \xi}(x, \nabla S)\right) S_{x x}=0 .
$$

Generally, the velocity field calculated from the Hamiltonian system has a jump simultaneously with a jump in $p=\nabla S$ (and then in $\dot{x}$ ). Thus, the problem (which is still open!) is to solve the transport equation in a discontinuous velocity field. We avoid this problem by considering a squared solution of the transport equation $\rho=\varphi_{0}^{2}$. Madelung [11] (about 100 years ago!) noticed that it satisfies the continuity equation

$$
\rho_{t}+(\nabla, \dot{x} \rho)+\frac{\rho}{2} \operatorname{tr} P_{x \xi}=0
$$

in the smooth case.

Our case is more complicated, namely, we again have a discontinuous velocity field. There are a few approaches to the solution of this problem: the theory of measure solutions [12-17], the box approximation [18], the weak asymptotics method [19-22], the method of generalized characteristics [23-25]. The last approach allows one to construct a solution to the continuity equation in the case where the singular support of the velocity field is a stratified manifold with smooth strata which are transversal to the (incoming!) trajectories of the velocity field.

If the singular support of the velocity field preserves its structure on some time interval $\left[t_{1}, t_{2}\right]$ (the mapping of the singular support induced by a shift along the Hamiltonian flow is a diffeomorphism), then one can show that the singular support of the velocity field has the required structure. If the structure is changing (e.g., a jump appears see Figures 5 and 4, the last step of evolution with time), then one can use the weak asymptotics method to construct the global solution to the Hamilton-Jacobi and continuity equations. This approach is based on a "new method of (generalized) characteristics" constructed by Danilov and Mitrovic [23-25] in the case where the strata of the singular support are of codimension 1.

The main idea of this approach is to consider the singularity creation as the result of interaction of solitary nonlinear waves.
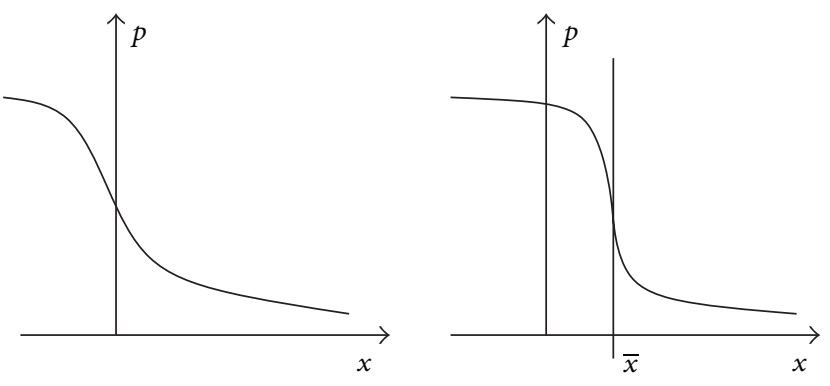

Figure 5

A simple example is the Hamiltonian flow corresponding to the heat equation in the previous example. The HamiltonJacobi equation in this case is equivalent to the Hopf equation for the momentum $p$ :

$$
p_{t}+\left(p_{x}\right)^{2}=0
$$

The solution in this example has the form

$$
\begin{aligned}
p= & P_{0} H\left(x-\phi_{1}\right)+P_{1} H\left(\phi_{2}-x\right) \\
& +a\left(H\left(\phi_{1}-x\right)\left(\phi_{1}-x\right)-H\left(\phi_{2}-x\right)\left(\phi_{2}-x\right)\right),
\end{aligned}
$$

and is plotted below in Figure 8 (here, $H$ is the Heaviside function, and $\left.a=\left(P_{0}\left(\phi_{1}, t\right)-P_{1}\left(\phi_{2}, t\right)\right) /\left(\phi_{2}-\phi_{1}\right)\right)$.

To consider $p^{2}$ we have to calculate the product

$$
H\left(\phi_{1}-x\right) H\left(\phi_{2}-x\right) .
$$

Here, the following equality holds:

$$
\begin{aligned}
H\left(\phi_{1}-x\right) H\left(\phi_{2}-x\right) & \\
= & B\left(\frac{\phi_{2}-\phi_{1}}{\mu}\right) H\left(\phi_{1}-x\right) \\
& +\left(1-B\left(\frac{\phi_{2}-\phi_{1}}{\mu}\right)\right) H\left(\phi_{2}-x\right)+O_{D^{\prime}}(\mu),
\end{aligned}
$$

where $\mu \rightarrow 0+0$ is a new small parameter and $O_{D^{\prime}}(\mu)$ is a small quantity in the sense of distributions,

$$
\left(O_{D^{\prime}}(\mu), \psi\right)=O(\mu),
$$

for each $\psi$ which is a test function. The time evolution of the function $p$ is such that the slanting intercept of the straight line preserves its shape until it takes vertical position, and then a jump propagates. This means that at every instant of time the solution anzatz can be presented in the form of a linear combination of Heaviside functions. This allows one to use a formula expressing the product of Heaviside functions as their linear combination up to a remainder, that is, small in the $D^{\prime}$-sense. Hence, we conclude that the functions $p$ and $p^{2}$ (the latter up to a small quantity) uniformly in-time belong to the same linear space; for detail, see [23-26]. This allows one to apply the same procedure as in [23-26] to solve this problem uniformly in-time. 
Taking $\phi_{1}-\phi_{2}=O\left(\varepsilon^{\delta}\right), 0<\delta<1$, at some initial instant of time and choosing an interval $\left[\phi_{2}, \phi\right]$ to include the point of overturning, we obtain a small correction to our Lagrangian manifold, that is, negligible as $\varepsilon \rightarrow 0$. But this correction allows one to apply the above technique for constructing the global-in-time solution describing the singularity appearance in the framework of the method of characteristics. In this framework we have new nonintersecting characteristics (Figure 7) instead of classical intersecting characteristics (Figure 6).

It remains to explain how we can go back from a generalized solution of the continuity equation to a solution of the transport equation. As was shown in [12,13,15-17] and in other papers listed above, the generalized solution to the continuity equation in this case has the form

$$
\rho=\rho_{\text {reg }}+E \cdot \delta_{\Gamma}
$$

where $\rho_{\text {reg }}$ is a smooth function outside $\Gamma, \Gamma$ is a stratified manifold (singular support) of $\rho$ coinciding with the singular support of $\nabla \phi, E$ is a function whose domain is $\Gamma$, and $\delta_{\Gamma}$ is the Dirac $\delta$-function on $\Gamma$. Using the algorithm given in [23-26], one does not obtain expression (31) but obtains its regularization, where the key point is the regularization of the $\delta$-function. Here, the following lemma can be used.

Lemma 5. Let $0 \leq \omega(x, t, z) \in C^{\infty}\left(\mathbb{R}^{m} \times[0, T] \times R^{1}\right)$, and let $\psi(x) \in C^{\infty},|\nabla \psi| \neq 0$. Let also the following estimate hold:

$$
\left|\frac{\partial^{|\alpha|+\beta} \omega(x, t, z)}{\partial x_{1}^{\alpha_{1}} \cdots \partial x_{m}^{\alpha_{m}} \partial z^{\beta}}\right| \leq C_{\alpha \beta}|z|^{-2 m-\varkappa},
$$

where $x>0$.

Then, in $D^{\prime}\left(\mathbb{R}^{m}\right)$ one has

(i) $\varepsilon \omega(x, t,(\psi(x)-t) / \varepsilon)=A(x, t) \delta(\psi(x)-t)+O_{D^{\prime}}(\varepsilon)$,

(ii) $\left[\varepsilon^{-1} \omega(x, t,(\psi(x)-t) / \varepsilon)\right]^{2}=O_{D^{\prime}}\left(\varepsilon^{1 / 2}\right)$.

Proof. Let $\psi_{x_{1}}^{\prime} \neq 0$. Then, we make the change of variables $\psi(x)-t=z \varepsilon, x_{2}=x_{2}, \ldots, x_{m}=x_{m}$, and for all test functions $\eta(x) \in C_{0}^{\infty}\left(\mathbb{R}^{m}\right)$ we obtain

$$
\begin{aligned}
\int_{\mathbb{R}^{m}} & \varepsilon^{-1} \omega\left(x, t, \frac{\psi(x)-t}{\varepsilon}\right) \eta(x) d x \\
& =\int \omega(x, t, z)\left(\psi_{x_{1}}^{\prime}\right)^{-1} \eta\left(x_{1}(z, \check{x}, \varepsilon), \check{x}\right) d z d \check{x},
\end{aligned}
$$

where $\check{x}=\left(x_{2}, \ldots, x_{m}\right)$. The last integral converges due to (32), and we obtain the first statement of the lemma with

$$
A(x, t)=\int_{\{\psi(x)=t\}} \int_{\mathbb{R}^{1}} \omega(x, t, z) d \nu(x) d z,
$$

where $d v(x)=\left|\psi_{x}^{\prime}\right|^{-1} d x$ is the Leray measure on the surface $\{\psi(x)=t\}$. The general case can be considered similarly (also see $[21])$.

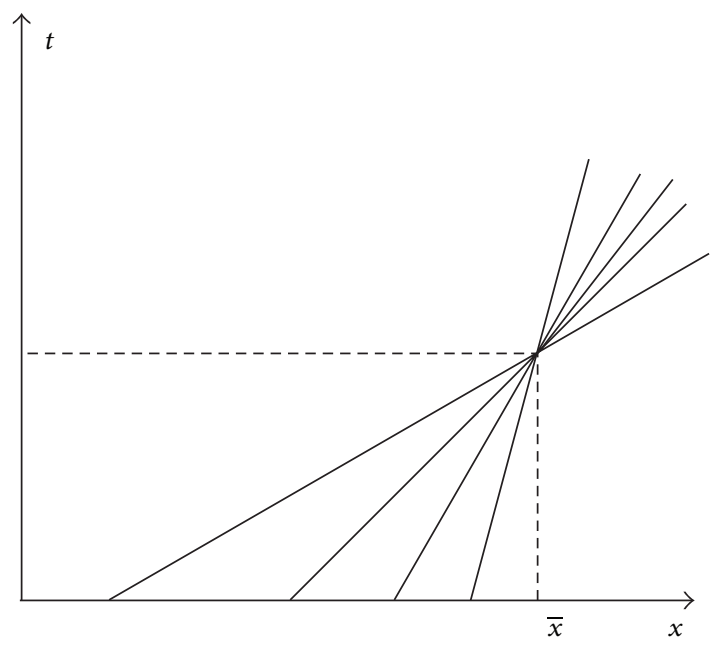

Figure 6: Classical characteristics.

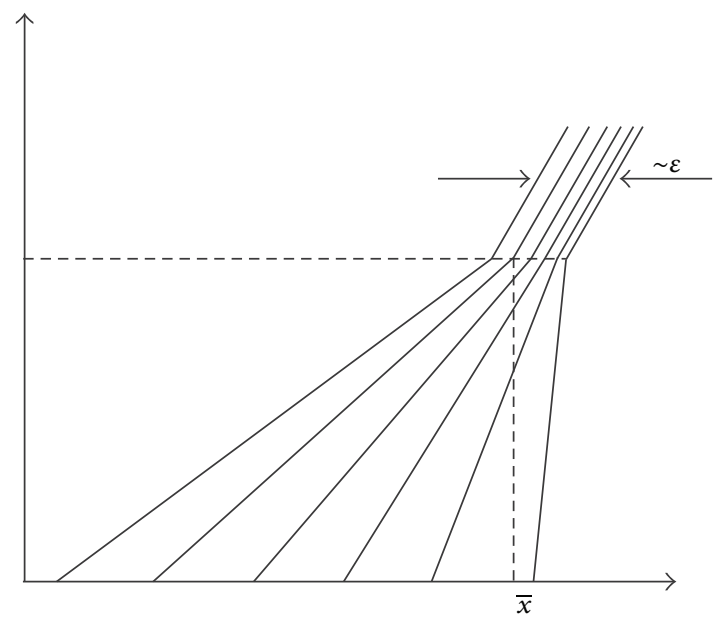

Figure 7: New characteristics.

Using the same procedure for item (ii), we obtain

$$
\begin{aligned}
& \int \varepsilon^{-1 / 2} \sqrt{\omega\left(x, t, \frac{\psi(x)-t}{\varepsilon}\right)} \eta(x) d x \\
& =\varepsilon^{1 / 2} \int \sqrt{\omega(x, t, z)} \psi_{x_{1}}^{-1} \eta\left(x_{1}(z, \check{x}, \varepsilon), \check{x}\right) d \check{x} .
\end{aligned}
$$

Hence, the proof of the lemma is complete.

Now, we can consider the case of stratified manifold.

Each $k$ th stratum can be presented as a level surface for some function $\psi_{k}(x)$, and we can use the statement of the above lemma for each stratum.

Here, the last remark concerns expression (31). We have

$$
\begin{array}{r}
\sqrt{\rho_{\text {reg }}+\varepsilon^{-1} \omega\left(x, t, \frac{\psi(x)-t}{\varepsilon}\right)}-\sqrt{\rho_{\text {reg }}} \\
=\frac{\varepsilon^{1 / 2} \omega(x, t,(\psi(x)-t) / \varepsilon)}{\sqrt{2 \varepsilon \rho_{\text {reg }}+\omega(x, t,(\psi(x)-t) / \varepsilon)}},
\end{array}
$$




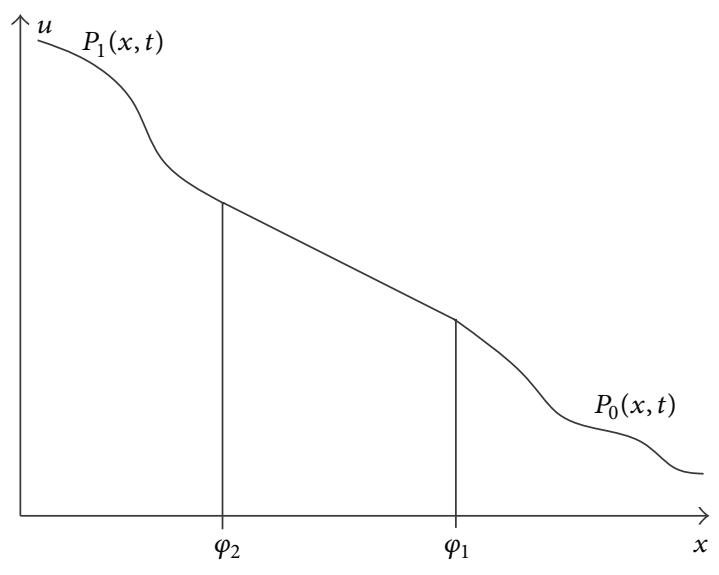

FIGURE 8: Plot of the function $p$.

and the above considerations lead to the following conclusion:

$$
\sqrt{\rho_{\text {reg }}+\varepsilon^{-1} \omega(x, t,(\psi(x)-t) / \varepsilon)}=\sqrt{\rho_{\text {reg }}}+O_{D^{\prime}}\left(\varepsilon^{1 / 2}\right)
$$

or in the limit form and in the weak sense to

$$
\sqrt{\rho_{\text {reg }}+E \delta_{\Gamma}}=\sqrt{\rho_{\text {reg }}} .
$$

Thus, we can prove the following theorem.

Theorem 6. Suppose that the following conditions are satisfied for $t \in[0, T], T>0$ :

(1) there exists a smooth solution of the Hamiltonian system,

(2) the singularities of the velocity field

$$
u=\nabla_{\xi} P(x, \nabla S)
$$

form a stratified manifold with smooth strata and $\operatorname{Hess}_{\xi} P(x, \xi)>0$.

Then, there exists a generalized solution $\rho$ of the Cauchy problem for the continuity equation in the sense of the integral identity introduced in [21,22], and at the points where the projection $\pi$ is bijective, the asymptotic solution of the Cauchy problem for Kolmogorov-Feller-type equation has the form

$$
u=\exp \left(-\frac{S(x, t)}{\varepsilon}\right)\left(\sqrt{\rho_{\text {reg }}}+O(\varepsilon)\right)
$$

\section{Backward-in-Time Motion}

As was shown above, all that we need to go forward-in-time is the Hamiltonian system:

$$
\begin{array}{cc}
\dot{x}=\nabla_{\xi} P(x, p), & \left.x\right|_{t=0}=\alpha, \\
\dot{p}=-\nabla_{x} P(x, p), & \left.p\right|_{t=0}=p(\alpha) .
\end{array}
$$

We change the time direction as $t \rightarrow-t$, and then we have

$$
\begin{aligned}
-\dot{X} & =\nabla_{\xi} P(x, \Xi), \\
-\dot{\Xi} & =-\nabla_{x} P(x, \Xi) .
\end{aligned}
$$

We want to solve the inverse problem:

$$
X(\alpha, 0)=x(\alpha, T), \quad \Xi(\alpha, 0)=p(\alpha, T) .
$$

The right-hand sides are considered as given data, and we are looking for $X(\alpha, t)$ and $\Xi(\alpha, t)$ for $0 \leq t \leq T$. Obviously, in our case, the solutions have the form

$$
X(\alpha, t)=x(\alpha, T-t), \quad \Xi(\alpha, t)=p(\alpha, T-t) .
$$

We draw the following conclusion: the "same" trajectories can be used to move forward and backward in time. But the incoming trajectories become outcoming ones, and vice versa.

\section{Corollary 7. Stable jumps become unstable.}

But if there are no jumps (no singularities of the projection mapping $\pi: \Lambda_{n}^{t} \rightarrow \mathbb{R}_{x}^{n}$ ), then our geometry (and the asymptotic solution!) is invertible in-time. This means that if we take the Cauchy problem solution $u$ for the parabolic PDE such that

$$
\left.u\right|_{t=0}=\exp \left(-\frac{S_{0}(x)}{\varepsilon}\right) \varphi^{0}(x),
$$

then the leading term of the asymptotic solution for $t=T$ has the "WKB" form

$$
\left.u_{\mathrm{as}}\right|_{t=T}=\exp \left(-\frac{S(x, T)}{\varepsilon}\right) \varphi_{0}(x, T) .
$$

Then, taking the last function as the initial data for the parabolic PDE in the inverse time (let $v_{\mathrm{as}}(x, t)$ be its asymptotic solution), we obtain (recall that $T$ is an instant of inverse time)

$$
\begin{aligned}
v_{\mathrm{as}}(x, T) & =\exp \left(-\frac{S_{0}(x)}{\varepsilon}\right) \varphi^{0}(x)(1+0(\varepsilon)) \\
& =\left.u\right|_{t=0}(1+O(\varepsilon)) .
\end{aligned}
$$

And surely these initial data will give the same leading term of the asymptotic solution for $t=0$. We want to stress once again that this statement is true if the projection mapping $\pi$ : $\Lambda_{n}^{t} \rightarrow \mathbb{R}_{x}^{n}$ has no singularities. But a jump brings problems.

By "measurements" one cannot recognize a multivalued function $S(x, t)$ (see Figure 3 and (17)). It is possible to "see" only the function $\Phi(x, t)=\min S(x, t)$, and thus only a Lagrangian manifold with a jump can be determined. Of course, one can try to make a smooth regularization of the jump like it is shown in Figure 9 (the left-hand side) with equal squares of dashed areas, but obviously such a regularization is not unique. It is interesting to note that if one uses a manifold with a jump, then the problem of backwardin-time motion becomes similar to the well-known problem of unstable jump decay. 


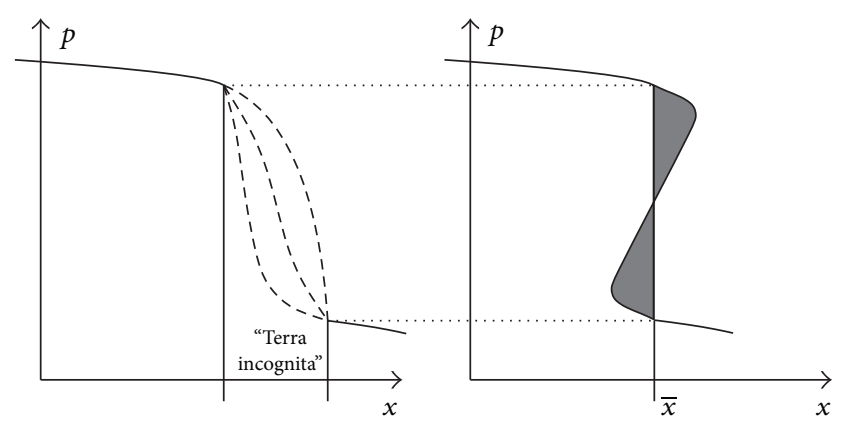

FIGURE 9

By reconstruction of the Lagrangian manifold, we mean the following. We choose an approximation of a jump by a smooth curve and then move the whole curve (Lagrangian manifold) backward-in-time along characteristics (42). After that if the projection map to $x$-axis is not unique, we form a jump as a vertical segment with the condition of equal squares; see, for example, Figure 4 . The last rule means that the action function corresponding to the shifted manifold is continuous. This property can be used to construct a Lagrangian manifold shifted backward-in-time in a multidimensional case, but we do not explain this here.

It is clear that all points of the Lagrangian manifold shifted backwards which are not the shifted points of a regularization curve (do not lie under the "terra incognita") coincide with the points of the initial Lagrangian manifold corresponding to generalized WKB solution (if such a solution exists) at $t=T$. Generally speaking, the one-to-one correspondence between the global-in-time generalized WKB solutions (more precisely, their logarithmic week limits) and the Lagrangian manifolds in the form that we are using does not hold. But we will assume that we are working with a subset of generalized WKB solutions which uniquely correspond to stratified Lagrangian manifolds globally in-time. Under this assumption and previous explanations we can uniquely reconstruct the Lagrangian manifold and the leading term of corresponding generalized WKB solution outside the "terra incognita."

But fortunately we can move ahead using the weak sense considerations. The main point is that it may happen that the function $S$ does not attain its minimum (maximum) inside the "terra incognita." This allows one to calculate the integrals containing the reconstructed solution without taking the "terra incognita" into account in the case where the integrand support contains this "terra incognita", and we can formulate the following statement.

Theorem 8. Let the symbol $P(x, \xi)$ defined by (2) be such that the function $B$ and the measure $\mu$ do not depend on $x$ and $V=0$. Let $u_{\varepsilon}(x, t)$ be a solution of the Cauchy problem for a Kolmogorov-Feller-type equation. Suppose that, for some $t \in(0, T)$, the logarithmic limit (action function) $S(x, t)=$ $\lim _{\varepsilon \rightarrow 0}\left(-\varepsilon \ln u_{\varepsilon}\right)$ and the generalized amplitude $\rho=w-$ $\lim _{\varepsilon \rightarrow 0} \exp (2 S / \varepsilon) u_{\varepsilon}^{2}$ exist.
Let the singular support of $S(x, t)$ be a stratified manifold $\Gamma, \rho$, has the form (31), and, $\rho_{\mathrm{reg}}$ be the regular part of the generalized solution to the continuity equation in the sense of distributions. Then, for an arbitrary test function $\phi=\phi(x)>0$ from the Schwartz space and for all $t \in[0, T]$, the limit as $\varepsilon \rightarrow 0$ of the integral

$$
C \int\left(u_{\varepsilon}-\rho_{\text {reg }}^{1 / 2} \exp \left(-\frac{S}{\varepsilon}\right)\right) \phi d x
$$

is equal to 0 , where $C^{-1}=\int u_{\varepsilon} \phi d x$.

In fact, these statements mean that from the viewpoint of the weak sense (momentum), the density reconstructed outside the "terra incognita" can be used in the same manner as the leading term of the asymptotic solution constructed earlier by Maslov's tunnel canonical operator and its modifications, [1-6].

Before we explain how to prove this statement, we briefly recall the essence of the Laplace method which is the main tool for investigating the integrals considered in the theorem.

This approach was developed to calculate integrals of the form

$$
I(\varepsilon)=\int_{\Omega} f(x) e^{S(x) / \varepsilon} d x,
$$

where $\Omega$ is either a closed domain in $\mathbb{R}^{n}$ or an interval. The leading term of $I(\varepsilon)$ as $\varepsilon \rightarrow 0+0$ is the sum of terms corresponding to the points at which the function $S(x)$ attains its minimal values.

For $n=1$, one has either

(1) $I(\varepsilon)=\varepsilon\left((f(a)+O(\varepsilon)) / S^{\prime}(a)\right) e^{-S(a) / \varepsilon}$, where $\Omega=[c$, d] and $\min _{x \in \Omega} S(x)=S(a), S^{\prime}(a) \neq 0$,

or

(2) $I(\varepsilon)=\sqrt{2 \pi \varepsilon / S^{\prime \prime}(a)}[f(a)+O(\varepsilon)] e^{S(a) / \varepsilon}$, where $a \in(c$, d), $\min _{x \in \Omega} S(x)=S(a)$.

If $a$ is an interior point of $\Omega \in \mathbb{R}^{n}$, then

$$
I(\varepsilon)=(2 \pi \varepsilon)^{n / 2}\left[\operatorname{det} S_{x x}^{\prime \prime}(a)\right]^{-1 / 2}[f(a)+O(\varepsilon)] e^{-S(a) / \varepsilon} .
$$

Below, we consider the case where (for one spatial variable) the point $x=a$ of the function $S$ minimal value is an interior point of the segment $(c, d)$, but the functions $S$ and $f$ are only piecewise continuous with jumps of derivatives (for $S$ ) and with jumps of the function $f$ and its derivatives at $x=a$. In this case, formula (1) should be modified as

$$
I(\varepsilon)=\exp \left(-\frac{S(a)}{\varepsilon}\right)\left[\frac{f_{+}(a)}{S_{+}^{\prime}(a)}+\frac{f_{-}(a)}{S_{-}^{\prime}(a)}\right]+O(\varepsilon),
$$

where $f_{ \pm}(a)$ and $S_{ \pm}^{\prime}(a)$ are the values of corresponding functions and derivatives from the right $(-)$ and from the left $(-)$.

Other possible situations including the cases where the above-listed derivatives of the function $S$ can be equal to zero are described in $[27,28]$. 
Any way, the above considerations lead to the following conclusion: under the assumption that the minimum of $S$ is attained on the boundary of the domain of integration, the value of the integral (with a high accuracy) depends only on the boundary values of the integrand (and its derivatives as well).

Now, I will briefly outline the proofs of the above statement. about invertibility in time. It can be divided in two parts. The first is to prove that, for all smooth reconstructions of the Lagrangian manifold in the "terra incognita" domain; the corresponding function $S_{0}=S_{0}(x)$ (for some fixed $t$ ) cannot attain its minimum value inside this domain, see the lemma below. This allows one to use the Laplace method to calculate the integrals mentioned in that statements taking into account that, due to this method and the lemma, the results of these calculations do not depend on the integrand values inside the "terra incognita" domain. The proof is finished by taking account of the estimate

$$
u_{\mathrm{as}}-u=O(\varepsilon) u_{\mathrm{as}}
$$

which is true outside the singular support of the function $S$; see the theorem above.

We will concentrate our efforts on one-dimensional considerations and the case with the symbol $P(\xi, x)=P(\xi)$.

Now, I formulate the lemma as follows.

Lemma 9. Let the symbol $P(x, \xi)$ defined by (2) be such that the function $B$ and the measure $\mu$ do not depend on $x$. Then the function $S$, which is the action function corresponding to the Lagrangian manifold, cannot attain the minimal value inside the "terra incognita" domains.

We begin our consideration with a special case where the operator symbol $P(x, \xi)$ does not depend on $x$ and restrict ourselves to the study of the one-dimensional case. Let $(a, b)$ be an interval inside the "terra incognita" domain, and let $\bar{x}_{0} \in$ $(a, b)$. We prove this by contradiction. First, we describe the "terra incognita" domain. It is easy to see that the upper end of the jump segment moves with horizontal speed $-P_{\xi}\left(p_{+}\right)$ and the lower end moves with the speed $-P_{\xi}\left(p_{-}\right)$, where $p_{ \pm}$ are $p$-coordinates of the upper $(+)$ and lower $(-)$ ends. At the same time, the horizontal speed of a jump is

$$
\phi_{t}=-\frac{P_{\xi}\left(p_{+}\right)-P_{\xi}\left(p_{-}\right)}{p_{+}-p_{-}} .
$$

By the assumption $P_{\xi \xi}>0$, we immediately obtain

$$
-P_{\xi}\left(p_{+}\right)<\phi_{t}<-P_{\xi}\left(p_{-}\right) .
$$

The last inequality describes the "terra incognita," which is a set of points between projections of the jump upper and lower ends shifted backwards. Let us consider some instant of time $t^{-} \in(0, T)$ and take the obtained manifold as the initial one. It is clear that for each projection of the trajectory starting inside the "terra incognita," there is another (at least one) projection of the trajectory starting inside the "terra incognita" which intersects with the first one. It follows from the picture in Figure 9 that each point on the boundary of the dashed domain has at least another one with the same projection on the $x$-axis. This means that along each projection of the trajectory starting inside the "terra incognita," the Jacobian $\partial x / \partial x_{0}$ is equal to zero at some instant of time.

Assume that the function $S$ attains its minimal value at the point $\bar{x}_{0} \in(a, b)((a, b)$ is a "terra incognita" domain). We prove that the following inequality is true along the trajectory of the Hamiltonian system whose projection starts at $\bar{x}_{0} \in$ $(a, b)$ :

$$
\left.\frac{D x}{D x_{0}}\right|_{x_{0}=\bar{x}_{0}} \neq 0
$$

This inequality leads to contradiction because, by assumption, $\bar{x}_{0}$ belongs to the "terra incognita" domain, and hence it belongs to the projection of the image of the singular (vertical) part of the Lagrangian manifold under a backwardin-time shift along the trajectories of the Hamiltonian system. In turn, this means that the projections of all trajectories such that the starting points of their projections belong to the "terra incognita" must intersect at a point in the case of the forward-in-time motion. So, the above inequality, leads to a contradiction. To prove this inequality let us write the projection of the Hamiltonian system trajectory starting at $\bar{x}_{0}$. It has the form

$$
x=\bar{x}_{0}+t P_{\xi}\left(p_{0}\right), \quad p_{0}=S_{0 x_{0}}\left(\bar{x}_{0}\right) .
$$

It is clear that $p_{0}=S_{0 x_{0}}\left(\bar{x}_{0}\right)=0$. Thus,

$$
\left.\frac{\partial x}{\partial x_{0}}\right|_{x_{0}=\bar{x}_{0}}=1+t P_{\xi \xi}(0) S_{0 x_{0} x_{0}}\left(\bar{x}_{0}\right) \text {. }
$$

Taking into account the fact that

$$
\frac{\partial^{2} P}{\partial \xi^{2}}>0
$$

because of convexity of $P$ and

$$
\frac{\partial^{2} S_{0}}{\partial x_{0}^{2}} \geq 0
$$

due to the assumption that $\bar{x}_{0}$ is a point of minimal value, we obtain the required inequality (55). The multidimensional case differs from this case in that the scalar values $P_{\xi}$ and $S_{0 x_{0}}$ must be replaced by vectors (gradients). In turn, this leads to matrix inequality in (58) and (59). The problem is to derive that the eigenvalues of the matrix $P_{\xi \xi}(0) S_{0 x_{0} x_{0}}\left(\bar{x}_{0}\right)$ are nonnegative by using (58) and (59). For this, we can make a change of variables that reduces the matrix $S_{0 x_{0} x_{0}}\left(\bar{x}_{0}\right)$ to diagonal form. This transformation induces the corresponding transformation in the $p$-plane that transforms the matrix $P_{p p}(0)$ into a new symmetric positive matrix. Now, we note that the principal minors of the new matrix product are products of matrix-factor principal minors (because the second has diagonal form). The determinants of the matrixfactor principal minors are nonnegative, so the spectrum of the matrix $P_{\xi \xi}(0) S_{0 x_{0} x_{0}}\left(\bar{x}_{0}\right)$ is also nonnegative, and we again obtain (55). To finish our consideration, we have to investigate 
the case, where the symbol $P=P(x, \xi)$ depends on $x$. We again will stay at the point $\bar{x}_{0}$, where the function $S_{0}$ attains its minimum. If so, then

$$
\left.p\right|_{t=0}=\left.\frac{\partial S_{0}\left(x_{0}\right)}{\partial x_{0}}\right|_{x_{0}=\bar{x}_{0}}=0,
$$

and by assumptions, $p=0$ for $t>0$.

The system for the matrices $\partial x / \partial x_{0}$ and $\partial p / \partial x_{0}$ follows from the Hamiltonian system and has the form

$$
\begin{aligned}
& \frac{d}{d t} \frac{\partial x}{\partial x_{0}}=\frac{\partial^{2} P}{\partial \xi \partial x} \frac{\partial x}{\partial x_{0}}+\frac{\partial^{2} P}{\partial \xi^{2}} \frac{\partial p}{\partial x_{0}} \\
& \frac{d}{d t} \frac{\partial p}{\partial x_{0}}=\frac{\partial^{2} P}{\partial x \partial \xi} \frac{\partial p}{\partial x_{0}}+\frac{\partial^{2} P}{\partial x^{2}} \frac{\partial x}{\partial x_{0}}
\end{aligned}
$$

Because of our assumptions (see the statement of the lemma), we have $\left.P\right|_{\xi=0}=0,\left.P_{x x}\right|_{\xi=0}=0$, and $\left.P_{\xi x}\right|_{\xi=0}=0$. This means that, along the Hamiltonian system trajectory starting from the point $x=\bar{x}_{0}, p=0$, we have $p=0$ and $(d / d t)\left(\partial p / \partial x_{0}\right)=$ 0 . Thus, along the trajectory mentioned above, (61) and (62) have the form

$$
\begin{gathered}
\frac{d}{d t} \frac{\partial x}{\partial x_{0}}=\frac{\partial^{2} P\left(\bar{x}_{0}, 0\right)}{\partial \xi^{2}} \frac{\partial p}{\partial x_{0}} \\
\frac{d}{d t} \frac{\partial p}{\partial x_{0}}=0
\end{gathered}
$$

Integrating with respect to $t$, we can transform the first equation to the form of (56) and apply all above arguments concerning this equality. This ends the proof.

The statement of the lemma can be generalized as follows. Let $B$ be a linear function in $x$,

$$
\langle B, \xi\rangle=\sum_{k=1}^{n} b_{k} x_{k} \xi_{k} .
$$

Then, instead of (64), we obtain

$$
\frac{d}{d t} \frac{\partial p}{\partial x_{0}}=\frac{\partial B}{\partial x} \frac{\partial p}{\partial x_{0}},
$$

where the matrix $\partial B / \partial x$ has diagonal form with elements equal to constants.

Then,

$$
\begin{gathered}
\frac{\partial p}{\partial x_{0}}=\left.e^{t(\partial B / \partial x)} \frac{\partial p_{0}}{\partial x_{0}}\right|_{t=0}, \\
\frac{\partial x}{\partial x_{0}}=E+\left.\frac{\partial^{2} P}{\partial \xi^{2}} \int_{0}^{t} e^{t^{\prime}\left(\partial B / \partial x_{0}\right)} d t^{\prime} \cdot \frac{\partial p_{0}}{\partial x_{0}}\right|_{t=0} \\
=\int_{0}^{t} e^{t^{\prime}(\partial B / \partial x)} d t^{\prime} \\
\times\left(\left(\int_{0}^{t} e^{t^{\prime}(\partial B / \partial x)} d t^{\prime}\right)^{-1}+\left.\frac{\partial^{2} P}{\partial \xi^{2}} \frac{\partial p_{0}}{\partial x_{0}}\right|_{t=0}\right)
\end{gathered}
$$

$\geq 0$.
Here, we used the fact that the matrix

$$
\int_{0}^{t} e^{t^{\prime}(\partial B / \partial x)} d t^{\prime}
$$

has diagonal form with positive eigenvalues.

Thus, we came to the relation with the same properties as (56). One can do more and extend the statement for the case of arbitrary drift at least for the symbol of the form (2). But up to now, the presence of a potential destroys our picture, and I will further think about this.

\section{Acknowledgment}

This study was carried out within The National Research University Higher School of Economics Academic Fund Program in 2013-2014, research Grant no. 12-01-0082.

\section{References}

[1] V. P. Maslov, "Global exponential asymptotic behavior of solutions of the tunnel equations and the problem of large deviations," Trudy Matematicheskogo Instituta imeni V. A. Steklova, vol. 163, pp. 150-180, 1984 (Russian), International conference on analytical methods in number theory and analysis (Moscow, 1981).

[2] V. P. Maslov, Asymptotic Methods and Perturbation Theory, Nauka, Moscow, Russia, 1988.

[3] V. P. Maslov and V. E. Nazaĭkinskiŭ, "The tunnel canonical operator in thermodynamics," Funktsional'nyi Analiz $i$ ego Prilozheniya, vol. 40, no. 3, pp. 12-29, 2006.

[4] V. G. Danilov, "Asymptotics of nonoscillating fundamental solutions of $h$-pseudodifferential equations," Mathematical Notes, vol. 44, no. 3, pp. 406-409, 1988.

[5] V. G. Danilov and S. M. Frolovitchev, "Exact asymptotics of the density of the transition probability for discontinuous markov processes," Mathematische Nachrichten, vol. 215, pp. 55-90, 2000.

[6] V. G. Danilov, "A representation of the delta function via creation operators and Gaussian exponentials, and multiplicative fundamental solution asymptotics for some parabolic pseudodifferential equations," Russian Journal of Mathematical Physics, vol. 3, no. 1, pp. 25-40, 1995.

[7] S. Albeverio and V. G. Danilov, "Global in time solutions to Kolmogorov-Feller pseudodifferential equations with small parameter," Russian Journal of Mathematical Physics, vol. 18, no. 1, pp. 10-25, 2011.

[8] V. G. Danilov, "On singularities of continuity equation solutions," Nonlinear Analysis: Theory, Methods \& Applications, vol. 68, no. 6, pp. 1640-1651, 2008.

[9] Yu. Kifer, "On the asymptotic behavior of transition densities of processes with small diffusion," Theory of Probability and Its Applications, vol. 21, no. 3, pp. 527-536, 1976.

[10] V. Maslov and M. Fedoryuk, Semiclassical Approximation in Quantum Mechanics, Reidel, Dordrecht, The Netherlands, 1981.

[11] E. Madelung, "Quantentheorie in hydrodynamischer Form," Zeitschrift für Physik, vol. 40, no. 3-4, pp. 322-326, 1927.

[12] P. LeFloch, "An existence and uniqueness result for two nonstrictly hyperbolic systems," in Nonlinear Evolution Equations That Change Type, vol. 27 of IMA Vol. Math. Appl., pp. 126-138, Springer, New York, NY, USA, 1990. 
[13] G. Dal Maso, P. G. Lefloch, and F. Murat, "Definition and weak stability of nonconservative products," Journal de Mathématiques Pures et Appliquées, vol. 74, no. 6, pp. 483-548, 1995.

[14] H. Yang, "Riemann problems for a class of coupled hyperbolic systems of conservation laws," Journal of Differential Equations, vol. 159, no. 2, pp. 447-484, 1999.

[15] B. T. Hayes and P. G. LeFloch, "Measure solutions to a strictly hyperbolic system of conservation laws," Nonlinearity, vol. 9, no. 6, pp. 1547-1563, 1996.

[16] D. C. Tan, T. Zhang, T. Chang, and Y. X. Zheng, "Delta-Shock Waves as Limits of Vanishing Viscosity for Hyperbolic Systems of Conservation Laws," Journal of Differential Equations, vol. 112, no. 1, pp. 1-32, 1994.

[17] H. Yang, "Riemann problems for a class of coupled hyperbolic systems of conservation laws," Journal of Differential Equations, vol. 159, no. 2, pp. 447-484, 1999.

[18] M. Nedeljkov and M. Oberguggenberger, "Interactions of delta shock waves in a strictly hyperbolic system of conservation laws," Journal of Mathematical Analysis and Applications, vol. 344, no. 2, pp. 1143-1157, 2008.

[19] S. Albeverio and V. M. Shelkovich, "On the delta-shock front problem," in Analytical Approaches to Multidimensional Balance Laws, O. Rosanova, Ed., chapter 2, pp. 45-87, Nova Science Publishers, New York, NY, USA, 2006.

[20] V. G. Danilov and V. M. Shelkovich, "Propagation and interaction of shock waves of quasilinear equation," Nonlinear Studies, vol. 8, no. 1, pp. 135-169, 2001.

[21] V. G. Danilov and V. M. Shelkovich, "Dynamics of propagation and interaction of $\delta$-shock waves in conservation law systems," Journal of Differential Equations, vol. 211, no. 2, pp. 333-381, 2005.

[22] V. G. Danilov and V. M. Shelkovich, "Delta-shock wave type solution of hyperbolic systems of conservation laws," Quarterly of Applied Mathematics, vol. 63, no. 3, pp. 401-427, 2005.

[23] V. Danilov and D. Mitrovic, "Weak asymptotics of shock wave formation process," Nonlinear Analysis, Theory, Methods and Applications, vol. 61, no. 4, pp. 613-635, 2005.

[24] V. G. Danilov and D. Mitrovic, "Delta shock wave formation in the case of triangular hyperbolic system of conservation laws," Journal of Differential Equations, vol. 245, no. 12, pp. 3704-3734, 2008.

[25] V. G. Danilov and D. Mitrovic, "Shock wave formation process for a multidimensional scalar conservation law," Quarterly of Applied Mathematics, vol. 69, no. 4, pp. 613-634, 2011.

[26] V. G. Danilov, "Generalized solutions describing singularity interaction," International Journal of Mathematics and Mathematical Sciences, vol. 29, no. 8, pp. 481-494, 2002.

[27] F. W. J. Olver, Asymptotics and Special Functions, Computer Science and Applied Mathematics, Academic Press, New York, NY, USA, 1974.

[28] M. V. Fedoryuk, Saddle Point Method, Nauka, Moscow, Russia, 1977. 


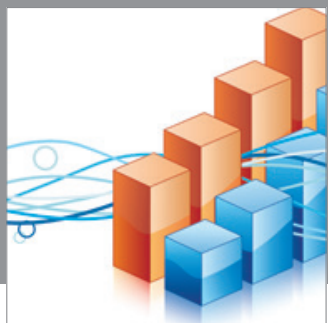

Advances in

Operations Research

mansans

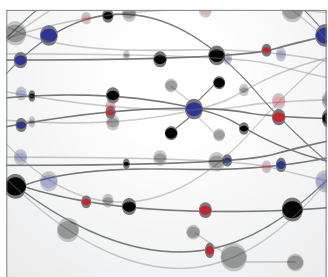

The Scientific World Journal
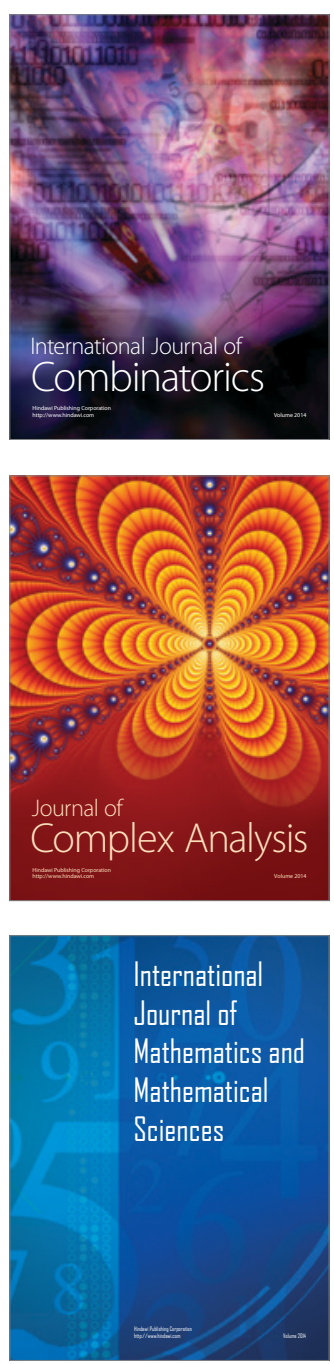
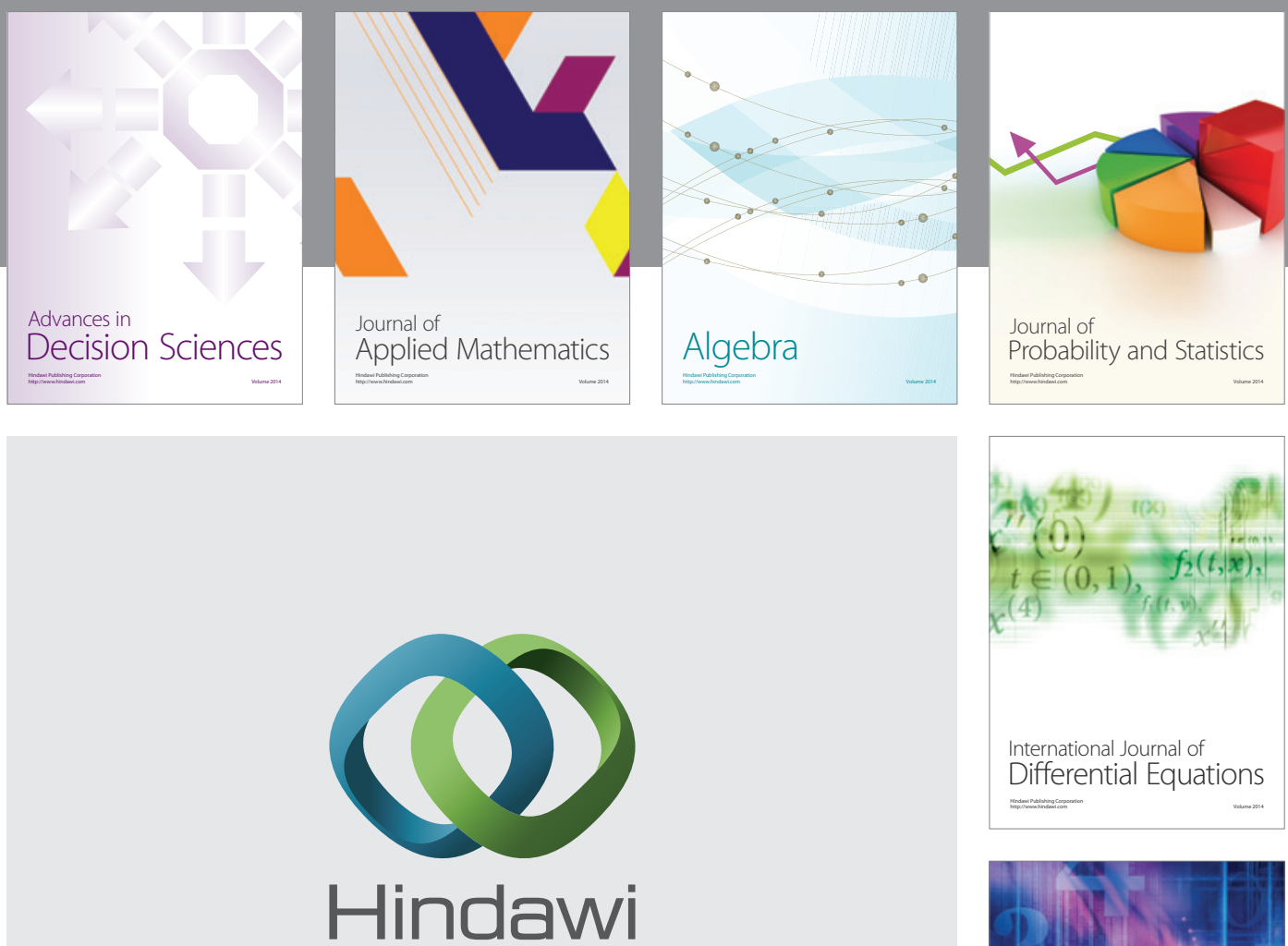

Submit your manuscripts at http://www.hindawi.com
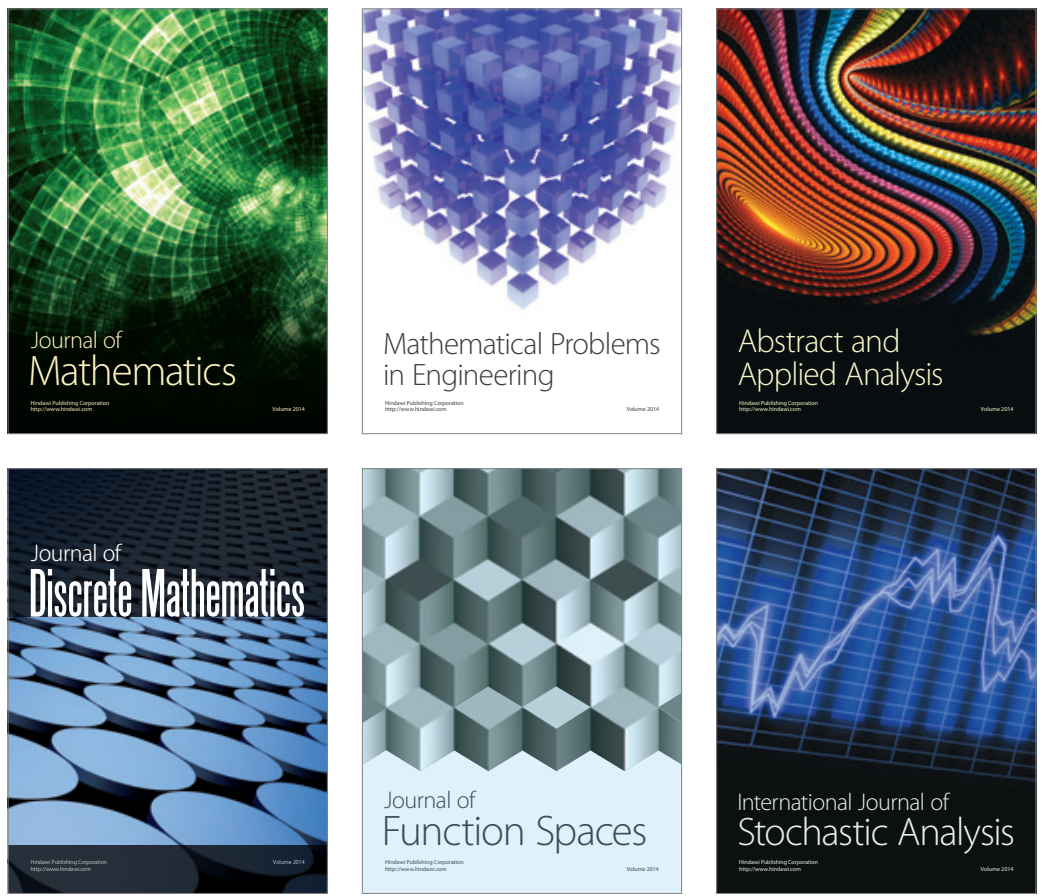

Journal of

Function Spaces

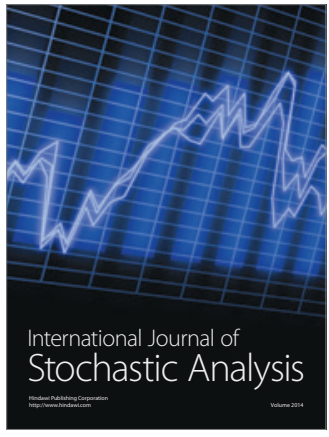

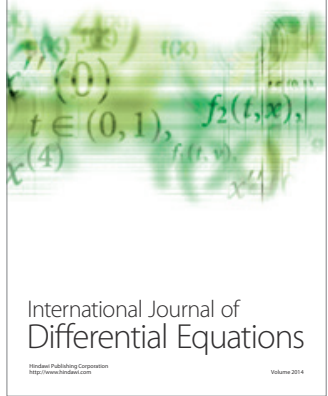
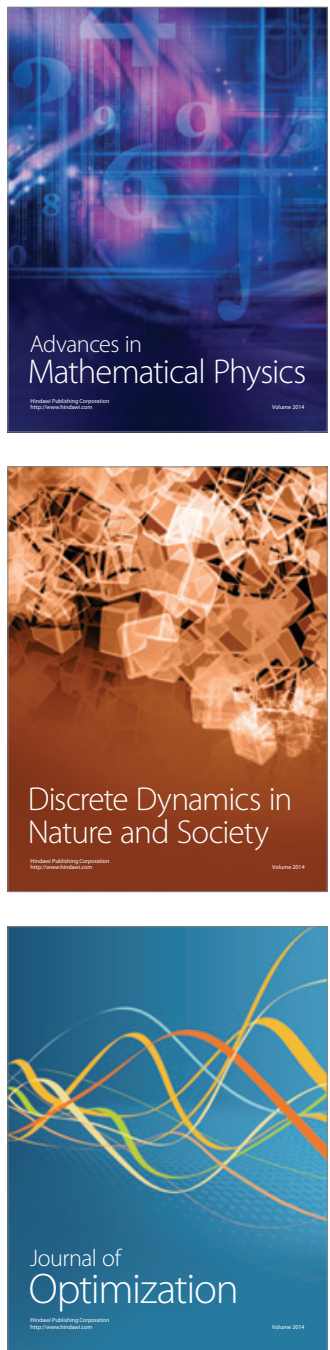